\title{
Belgeo
}

Revue belge de géographie

\section{L'art, un outil géographique pour mettre au jour et en œuvre la (dé)construction des espaces publics à Johannesburg (Afrique du Sud) : le cas de Mandela Square}

Art, a geographical tool to reveal and initiate the (un)making of public spaces in Johannesburg (South Africa): the Mandela Square case

\section{Pauline Guinard}

\section{(2)enEdition}

\section{Journals}

Édition électronique

URL : http://journals.openedition.org/belgeo/13305

DOI : 10.4000/belgeo.13305

ISSN : 2294-9135

\section{Éditeur :}

National Committee of Geography of Belgium, Société Royale Belge de Géographie

\section{Référence électronique}

Pauline Guinard, «L'art, un outil géographique pour mettre au jour et en œuvre la (dé)construction des espaces publics à Johannesburg (Afrique du Sud) : le cas de Mandela Square », Belgeo [En ligne], 3 | 2014, mis en ligne le 19 décembre 2014, consulté le 01 mai 2019. URL : http:// journals.openedition.org/belgeo/13305; DOI : 10.4000/belgeo.13305

Ce document a été généré automatiquement le 1 mai 2019.

Belgeo est mis à disposition selon les termes de la licence Creative Commons Attribution 4.0 International. 


\section{L'art, un outil géographique pour mettre au jour et en œuvre la (dé)construction des espaces publics à Johannesburg (Afrique du Sud) : le cas de Mandela Square}

Art, a geographical tool to reveal and initiate the (un)making of public spaces in Johannesburg (South Africa): the Mandela Square case

\section{Pauline Guinard}

\section{Introduction}

Examiner les espaces publics à Johannesburg, capitale économique de l'Afrique du Sud, semble à première vue une gageure puisque cette ville est précisément connue pour en être dépourvue. Et de fait, pour un Européen, l'impression qui se dégage de cette ville lorsqu'on la découvre pour la première fois est que les espaces publics y font défaut: les gens, ou du moins certains d'entre eux - généralement les Blancs, ainsi que les classes moyennes et aisées -, se déplacent majoritairement en voiture, et non à pied ; les rues, particulièrement celles du centre-ville, sont délaissées par ces mêmes populations la journée, et quasiment abandonnées de tous la nuit ; les lieux de mixité sociale et « raciale $^{1}$ » sont rares. A contrario, les centres commerciaux, souvent appréhendés dans les villes occidentales (Sorkin, 1992) et plus encore européennes comme des espaces publics factices (Ghorra-Gobin, 2001), comme des «pseudo » espaces publics (Goss, 1993), font figure de principaux espaces de côtoiement et de rencontre de l'altérité. Reste alors à savoir si cette impression d'un manque d'espaces publics correspond effectivement à la réalité johannesburgeoise telle qu'elle est vécue par ses producteurs, ses habitants et plus généralement ses usagers, et non uniquement par ses observateurs extérieurs. La perception d'un déficit d'espaces publics à Johannesburg découle-t-elle d'une absence 
véritable ou résulte-t-elle d'une inadaptation du regard et des catégories d'analyse au contexte considéré ?

La question mérite d'autant plus d'être posée que depuis la chute de l'apartheid (1994), et de manière accélérée depuis la fin des années 2000, l'art sous toutes ses formes (sculptures, murals ${ }^{2}$, performances, etc.) est utilisé par les différents acteurs de Johannesburg (entreprises privées, pouvoirs publics, artistes ou citadins) pour - selon leurs dires - réinventer et reconstruire ces espaces, mis à mal hier par l'apartheid et aujourd'hui par le néolibéralisme. Si cette tendance s'inscrit dans un mouvement plus vaste d'affirmation de la culture en général (Zukin, 1995), et de l'art en particulier (Miles, 1997), comme éléments à part entière des stratégies urbaines à travers le monde, elle correspond aussi à une transformation plus spécifique des fonctions qui sont assignées à l'art dans les villes sud-africaines. Dans un contexte post-apartheid, l'art est en effet vu à la fois comme un possible vecteur de démocratisation de la société sud-africaine, permettant à tout un chacun d'exprimer librement ses idées, et comme un potentiel facteur de (re)construction et de (ré)invention des villes au-delà des divisions sociospatiales héritées (Minty, 2006; Marschall, 2010a, 2010b). L'art n'acquerrait donc pas seulement une place accrue dans les espaces publics des villes sud-africaines comme Johannesburg, mais il serait en outre reconnu par les acteurs urbains pour ses effets potentiels sur ces espaces, leurs usagers et, à travers eux, sur la ville dans son ensemble. Dans quelle mesure l'art qui se déploie dans les espaces urbains peut-il véritablement les rendre publics? Par les fonctions qui lui sont attribuées, les discours et les pratiques qui lui sont attachés, en quoi cet art peut-il être, pour le géographe, un instrument d'appréhension et de compréhension de ce que sont ou peuvent être les espaces publics johannesburgeois?

3 A partir du cas de Johannesburg et plus spécifiquement de (Nelson) Mandela Square, place centrale d'un des plus importants centres commerciaux de la ville, nous montrerons que l'art peut être un outil géographique à part entière, permettant de mettre en évidence, de comprendre mais aussi d'initier les processus de construction ou de déconstruction des espaces publics. Parce que ce qui nous intéresse est de comprendre comment l'art peut modifier les rapports - tant immatériels que matériels, tant symboliques que physiques des différents publics aux différents espaces et de ces publics entre eux, nous nous appuierons sur des enquêtes qualitatives de type anthropologique (Olivier de Sardan, 1995). Menées à Mandela Square de mars 2010 à septembre 2011, ces enquêtes associent des séances régulières et répétées d'observation participative qui ont donné lieu à la rédaction de carnets de terrain, des entretiens avec les producteurs et les usagers de cet espace, une analyse des discours de ces mêmes acteurs et, enfin, des techniques issues des méthodologies visuelles telle la prise de photographies à des fins documentaires ou comme support d'entretiens (Rose, 2007). Nous examinerons ainsi l'assertion qui consiste à dire qu'il n'y a pas d'espaces publics à Johannesburg et tenterons de la dépasser en envisageant l'art comme un prisme d'étude privilégié de ces espaces. Puis, à travers une analyse qualitative de Mandela Square, nous mettrons en évidence que l'art notamment lorsqu'il est contextuel (Ardenne, 2002) et relationnel (Bourriaud, 1998), c'est-à-dire conçu pour entrer en résonance avec un lieu et ses publics, peut être un vecteur de construction du caractère public des espaces, y compris à Johannesburg. 


\section{L'art de saisir les espaces publics à Johannesburg}

\section{Les espaces publics à Johannesburg, une absence?}

Véhiculée par nombre d'observateurs extérieurs et même partagée par certains de ses usagers et habitants, l'idée généralement répandue à propos de Johannesburg est que c'est une ville sans espaces publics. Parce qu' « il n'y a tout simplement pas d'espace public digne de ce nom » (2003, p. 367), Johannesburg constituerait même pour Jacques Lévy un contre-modèle de ville. Trois principaux facteurs motivent le plus souvent cette analyse.

Tout d'abord, l'apartheid et ses conséquences socio-spatiales. L'apartheid et avant lui la colonisation et l'Union sud-africaine ont en effet tendu à démanteler le caractère public des espaces sud-africains (Houssay-Holzschuch et Teppo, 2009) en assignant à chaque «public » défini sur des critères raciaux et ethniques un espace de résidence, de travail, etc. La conception même des espaces publics comme lieux de libre côtoiement et de potentielle rencontre de personnes diverses qui ne se connaissent pas (Joseph, 1984, 1998) était fondamentalement contraire aux idéaux raciaux et racistes de l'apartheid. Pour les tenants de ce régime, les espaces publics se devaient d'être des lieux de séparation ou à défaut, quand la stricte séparation n'était pas possible, de hiérarchisation des publics. Le fait que les bancs "publics» du centre-ville étaient réservés aux seules populations blanches illustre ce point. L'espace était alors envisagé comme un instrument de division et de classification des populations. Or, comme l'a montré Philippe Gervais-Lambony (2003), si l'apartheid et ses lois iniques prennent officiellement fin en 1994, leurs conséquences sociales, politiques, économiques et spatiales ne disparaissent pas du jour au lendemain. Les ségrégations héritées et leurs recompositions actuelles continueraient de saper le caractère public des espaces.

6 Ensuite, l'avènement de la ville néolibérale. La fin de l'apartheid s'est traduite par le choix d'un modèle de développement - notamment urbain - néolibéral. En termes d'espaces publics, cela se traduit par leur progressive privatisation. A partir des villes européennes, Christian Dessouroux (2003) distingue à ce sujet deux grandes logiques de privatisation que l'on retrouve dans les villes américaines comme sud-africaines :

- une appropriation par des acteurs privés d'espaces publics existants, que ce soit par la mise en place de partenariats public-privé, l'installation de systèmes de vidéosurveillance ou bien encore la fermeture de quartiers (Zukin, 1995 ; Fyfe et Bannister, 1996 ; Davis, 1997 [1990] ; Kohn, 2004 ; Dorier-Apprill et al., 2007 ; Bénit-Gbaffou et al., 2009) ;

- une appropriation de l'idée même d'espaces publics par des instances privées, se traduisant par la création d'« espaces privés ouverts au public » (Ghorra-Gobin, 2001), dont les centres commerciaux et les parcs de loisirs sont les figures types (Sorkin, 1992; Goss, 1993 ; Chivallon et al., 1998 ; Staeheli et Mitchell, 2006).

Plus encore, Marianne Morange (2011) montre que dans le cadre de la mise en place d'un régime néolibéral - compris au sens foucaldien comme une rationalité consistant à diffuser dans l'ensemble de la société les valeurs qui fondent le libéralisme (individualisme, compétitivité, etc.) - dans les villes sud-africaines, ce ne sont plus seulement les acteurs privés mais aussi les acteurs publics qui, en intériorisant les normes du privé, promeuvent la privatisation des espaces. Les espaces publics sud-africains seraient donc de moins en moins publics du fait du rôle croissant du privé dans leur gestion et conception. 
Enfin, la violence et la peur. Parce qu'elle s'accompagne le plus souvent d'un phénomène de sécurisation, la privatisation des espaces publics est accentuée à Johannesburg du fait de taux de criminalité élevés et d'un fort sentiment d'insécurité ${ }^{3}$ qui structure les pratiques des citadins et institue bien souvent la mise en sécurité des espaces en un préalable à toute rencontre avec l'autre (Dirsuweit, 2002 ; Dawson, 2005 ; Bremner, 2010). La multiplication des grilles, portes et autres systèmes d'auto-enfermement volontaire ne sont, à ce titre, rien d'autre qu'une matérialisation de cette peur qui parcourt et façonne la ville, tendant par là même à restreindre l'accès des populations à certains espaces publics réputés, à tort ou à raison, dangereux.

L'ensemble de ces facteurs (ségrégation, privatisation, sécurisation) contribuerait donc à nier le caractère public - la publicité au sens de publicness - des espaces. Pourtant, se réfère-t-on à la même vision des espaces publics lorsque l'on dénonce leur faible diversité socio-raciale, le poids du privé dans leur administration ou bien encore leur fermeture? Sont-ce, dans chaque cas, les mêmes espaces publics qui font défaut?

\section{Les espaces publics johannesburgeois : des espaces déconnectés}

10 La notion d'espaces publics recouvre une réalité sans doute plus hétérogène et polymorphe qu'il n'y paraît à première vue. L'hypothèse qui consiste à dire qu'il n'y a tout simplement pas d'espaces publics à Johannesburg, sans préciser plus avant ce qu'on entend par espaces publics, apparaît de ce fait rapidement réductrice et insatisfaisante. Pour mieux comprendre la réalité sud-africaine sans risquer de calquer une vision occidentale inadaptée au contexte (Navez-Bouchanine, 2001), pour mieux saisir ce qui change au-delà et en deçà des inerties structurelles qui modèlent les villes sud-africaines (Houssay-Holzschuch, 2010), nous avons cherché à établir une grille de lecture capable de rendre compte de ce que ne sont pas mais aussi de ce que sont les espaces publics johannesburgeois.

11 Des multiples appréhensions, définitions ou encore controverses visant à cerner rigoureusement ce que comprennent ces espaces dans la littérature francophone comme anglophone (voir notamment : Jacobs, 1977 ; Joseph, 1984, 1998 ; Sorkin, 1992 ; GhorraGobin, 2001 ; Mitchell, 2003 ; Berdoulay et al., 2004 ; Fleury, 2007 ; Lévy et Lussault, 2013), il est possible - comme l'ont mis en évidence Guénola Capron et Nadine Haschar-Noé (2007) d'une part et Myriam Houssay-Holzschuch (2010) d'autre part - de dégager trois acceptions des espaces publics :

- les espaces publics au sens juridique, soit des espaces relevant de la propriété publique ;

- les espaces publics au sens social, soit des espaces de rencontre et d'interaction avec l'autre ;

- l'espace public au sens politique, soit un possible espace de vie en société (sens faible) et/ou d'épanouissement de l'opinion et du débat publics (sens fort).

Bien évidemment, ces trois dimensions ne s'excluent pas mutuellement: elles se complètent, se chevauchent et entrent parfois en conflit. L'intérêt de cette grille de lecture réside néanmoins dans le fait qu'elle nous permet de relire la réalité johannesburgeoise sous un autre jour.

Prenons l'exemple des centres commerciaux. Parce qu'ils appartiennent à des acteurs privés, ce sont des espaces juridiquement privés. Dans le même temps et comme montré par Bruno Sabatier (2007), ces espaces sont aussi bien souvent - y compris en Afrique du Sud (Houssay-Holzschuch et Teppo, 2009) - des espaces de coprésence d'individus divers, 
ce qui en fait potentiellement des espaces socialement publics. A l'inverse, si les rues du centre-ville de Johannesburg relèvent bien de l'autorité publique métropolitaine, et sont donc juridiquement publiques, le fait qu'elles soient largement ségrégées met à mal leur caractère socialement public. Pour autant, cela n'empêche pas qu'y soient régulièrement organisées des manifestations qui contribuent à faire d'elles, du moins temporairement, des espaces politiquement publics, ce qui n'est qu'exceptionnellement le cas des centres commerciaux qui sont eux, à Johannesburg comme ailleurs (Mitchell et Staeheli, 2007), hautement régulés et contrôlés de façon à éviter ce type d'événement. Plus qu'à une absence, le sentiment d'un déficit d'espaces publics dans la métropole johannesburgeoise découlerait par conséquent d'une déconnexion de leurs différentes dimensions (juridique, sociale et politique): les espaces juridiquement publics ne s'identifient pas systématiquement aux espaces socialement publics, lesquels, à leur tour, ne correspondent pas nécessairement aux espaces politiquement publics.

Plus encore, le caractère public d'une des dimensions des espaces ne saurait être donné. De nombreux auteurs (voir notamment : Mitchell, 2003 ; Berdoulay et al., 2004 ; Capron et Haschar-Noé, 2007) ont mis en évidence le «mythe » des espaces publics comme réalité fixée une fois pour toutes quelles que soient les dimensions de la publicité considérées. Du fait de l'existence de mécanismes qui peuvent soit remettre en cause cette publicité (ségrégation, privatisation, etc.), soit la favoriser (diffusion d'un sentiment de sécurité, démocratisation, etc.), celle-ci n'est en effet jamais complètement acquise. Au contraire, la publicité est toujours à bâtir, à réaffirmer par les différents acteurs de ces espaces (usagers, autorités publiques, aménageurs, etc.). Dès lors, la question de savoir si un espace est public ou non constitue un faux problème ou du moins un problème mal posé. L'enjeu réside bien plutôt dans le degré de publicité de ces espaces et les modalités de construction de leur publicité. Il s'agit alors de saisir dans quelle mesure les processus à l'œuvre renforcent ou affaiblissent le caractère public des espaces, permettent de tendre vers cet idéal de publicité (publicisation) ou conduisent à s'en éloigner (privatisation). Une approche en termes de publicisation et de privatisation des espaces dans leurs dimensions juridique, sociale et politique, est ainsi essentielle pour ne pas tomber dans le piège d'une vision utopique des espaces publics, qui porterait irrésistiblement à conclure à leur absence. Reste à savoir s'il est possible de publiciser les espaces johannesburgeois et quel peut être le rôle de l'art dans ce processus.

\section{L'art, un prisme pour mettre à jour et en œuvre les processus de (dé)construction des espaces johannesburgeois}

Comme évoqué en introduction, il semble se dessiner depuis la fin de l'apartheid à Johannesburg un regain d'intérêt pour l'art, notamment lorsque celui-ci se déploie dans les espaces publics de la ville. Ce renouveau de l'art s'inscrit tout à la fois :

- dans un mouvement de changement du rôle attribué à l'art dans les sociétés et les villes contemporaines (Lacy, 1995 ; Miles, 1997 ; Hall et Robertson, 2001 ; Ruby, 2001 ; Sharp et al., 2005), sa valeur économique, sociale et politique tendant à prendre le pas sur sa valeur esthétique,

- et, de façon plus spécifique, dans une transformation des fonctions qui lui sont assignées en Afrique du Sud en général et dans les villes sud-africaines en particulier dont Johannesburg, l'art étant vu comme un élément à part entière de la reconstruction de la nation et de la métropole post-apartheid (Minty, 2006 ; Marschall, 2010a, 2010b). 
L'art qui se déploie dans les espaces publics urbains se voit donc progressivement attribuer des prérogatives nouvelles, et est d'ailleurs de plus en plus analysé comme tel par les géographes (voir notamment : Hawkins, 2011, 2013 ; Zebracki, 2012 ; Schuermans et al., 2012 ; Volvey, 2012 ; Grésillon, 2014). Ce nouvel art public serait potentiellement un art produit avec les espaces publics (Volvey, 2007, 2010), fait pour et par les publics de ces espaces (Lacy, 1995 ; Bourriaud, 1998 ; Zebracki, 2012), ou encore porteur et initiateur de débats publics (Lacy, 1995 ; Deutsche, 1998 ; Ruby, 2002 ; Vander Gucht, 2004 ; Van Essche, 2007). L'art ne serait pas simplement de plus en plus présent dans les espaces publics des villes comme Johannesburg mais aurait de surcroît des effets sur la publicité et la publicisation des espaces.

17 C'est en ce sens que l'art apparaît comme une clef de lecture pertinente des espaces publics, susceptible d'en révéler les multiples aspects. En effet, l'art qui est produit dans les espaces publics se rapporte potentiellement à toutes leurs dimensions : juridique, en tant qu'il est installé dans les espaces appartenant à la puissance publique ou à des acteurs privés ; sociale, en entendant favoriser la diversification des publics présents dans ces espaces et leur interaction; politique, en promouvant l'acceptation et l'expression de tous. Dans cette perspective, l'art est à envisager comme un prisme permettant de rendre visibles et de distinguer - comme le prisme au sens propre le fait pour les différents rayons qui composent la lumière - les différentes dimensions (juridique, sociale, politique) qui composent ces espaces. En outre, en prétendant agir sur les différentes dimensions de ces espaces, l'art pourrait également favoriser un mouvement de «reconnexion » de ces dimensions qui paraissent aujourd'hui particulièrement déconnectées à Johannesburg. Une telle approche ouvre une double perspective. D'une part, l'art permettrait de mieux saisir ce qu'est et peut être un espace public à Johannesburg, en mettant au jour les différentes dimensions de leur publicité. D'autre part, il pourrait participer à la (re)construction de leur caractère public, en renforçant l'une de ces dimensions éventuellement défaillante ou en faisant coïncider toutes les dimensions à la fois. L'art peut alors être envisagé comme un révélateur et un catalyseur des processus de construction ou de déconstruction du caractère public des espaces johannesburgeois.

\section{Mandela Square, un espace privé publicisé par l'art}

Pour mieux mettre en évidence les déconnexions qui affectent les différentes dimensions des espaces publics à Johannesburg ainsi que les mouvements de (dé)construction de chacune de ces dimensions qui peuvent être révélés ou initiés par l'art, nous avons choisi de nous pencher sur le cas de Mandela Square. Cette place nous intéresse à la fois parce que, bien que juridiquement privée, elle est - comme nous le montrerons - un des espaces les plus socialement divers de Johannesburg et parce qu'elle a été très précocement mise en art (figure 1). 
Figure 1. Mandela Square, un espace juridiquement privé ouvert au public et à l'art.
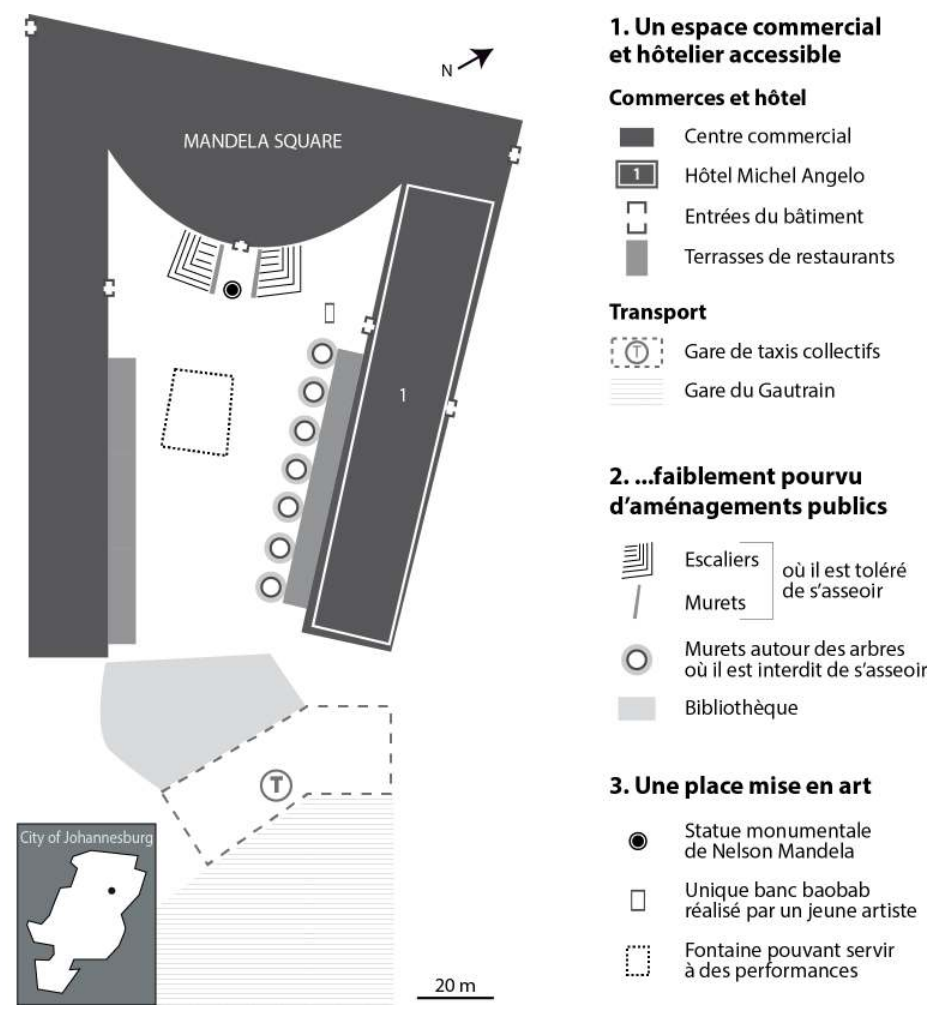

(C) P. GUINARD, 2014

\section{Mandela Square, un espace juridiquement privé mais socialement public}

En plein cœur d'un des plus prestigieux centres commerciaux de Sandton, qui est luimême un quartier emblématique du développement qu'ont pu connaître les riches banlieues blanches du nord de Johannesburg à partir des années 1960, et ce jusqu'à devenir, dans les années 1990, le nouveau centre économique de la métropole (Beavon, 2004), Mandela Square est réputé pour être un espace réservé à certaines catégories de populations (historiquement les populations blanches, aujourd'hui les populations aisées).

Pourtant, les observations et relevés que nous avons réalisés soulignent à quel point cette place est loin d'accueillir un public exclusivement blanc et/ou aisé. Pour appréhender la composition des publics présents dans cet espace, nous avons effectué au cours de la semaine du 7 au 13 mars 2011, un comptage systématique du nombre de personnes entrant et sortant de la place par une des portes les plus utilisées du centre commercial (y compris par des personnes ne se rendant pas nécessairement dans cet espace mais le traversant pour aller travailler), de façon à avoir un point de repère et à faciliter le dénombrement. Nous avons réalisé ce comptage toutes les cinq premières minutes de chaque heure, à la fois durant la semaine et le week-end, le jour comme la nuit (c'est-àdire après $18 \mathrm{~h}$ à cette période de l'année), en vue de mettre en évidence d'éventuelles variations dans le temps. Pour chaque personne dénombrée, nous tenions compte de sa couleur de peau, de son âge et de son genre (figure 2). 
Figure 2. Mandela Square, un espace socialement public?

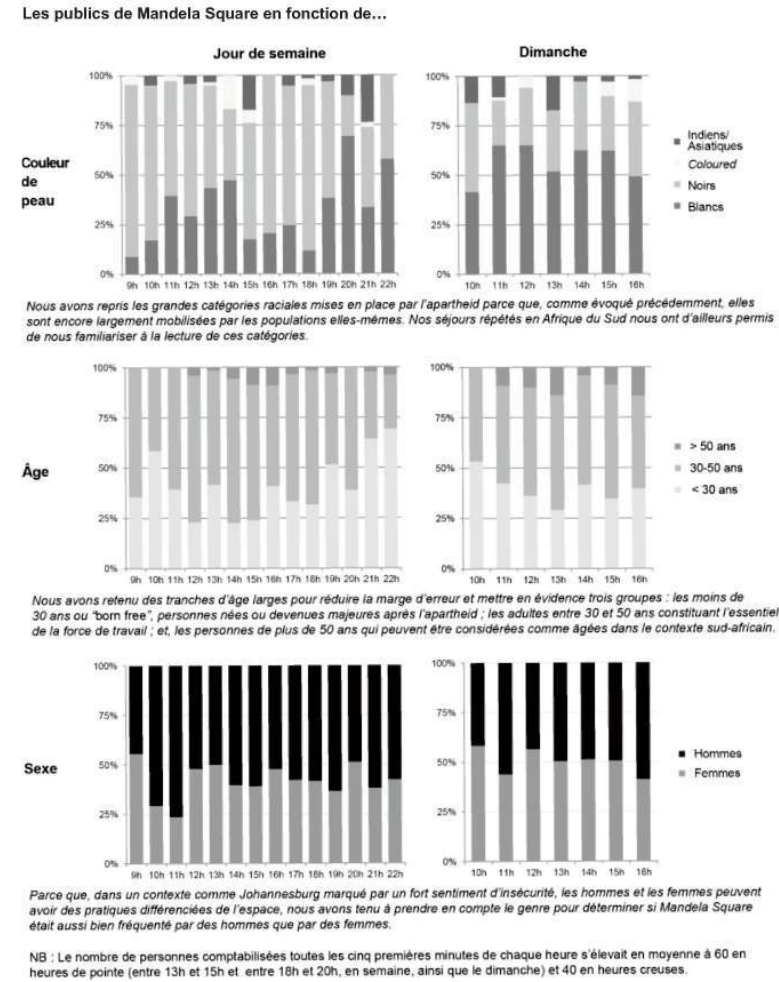

(C) P. GUINARD, 2014

Les usagers de Mandela Square sont divers aussi bien en ce qui concerne leur genre que leur âge et leur couleur de peau. Ce dernier point est particulièrement important.

En effet, cette relative mixité raciale qui pourrait être banale ailleurs ne l'est pas dans le contexte johannesburgeois qui reste marqué par la persistance de fortes ségrégations. La majorité des espaces ouverts au public et plus encore des espaces juridiquement publics de la métropole, tels ceux du centre-ville, étant encore largement ségrégés (Guinard, 2014), le simple fait que des personnes, indépendamment de leur couleur de peau, puissent être présentes de manière simultanée dans un même espace est somme toute exceptionnel. Par ailleurs, cette mixité raciale est en elle-même révélatrice d'une certaine pluralité quant à l'origine sociale et géographique des populations présentes, les critères de race, de classe sociale et de résidence étant historiquement et actuellement encore liés dans le contexte sud-africain où la ségrégation sociale se surimpose voire prend le pas sur la ségrégation raciale (Houssay-Holzschuch, 2010).

Que ce soit en termes d'âge, de genre, de couleur de peau ou de classe sociale, Mandela Square apparaît donc aujourd'hui comme un lieu d'exposition de la société sud-africaine dans sa diversité, comme un espace de coprésence des différents publics. Reste que, comme évoqué précédemment, pour qu'un espace soit socialement public cela suppose qu'il soit un lieu où des publics variés se trouvent et se rencontrent. L'art qui a lieu à Mandela Square peut-il alors être un vecteur non seulement de diversification des usagers mais aussi d'interaction entre ces derniers? 


\section{L'art, facteur de publicisation sociale et politique de Mandela Square} bronze de plus de six mètres de haut de Mandela qui y a été installée en mars 2004 (figure 3).

Figure 3. La statue de Mandela, facteur de publicisation de l'espace?

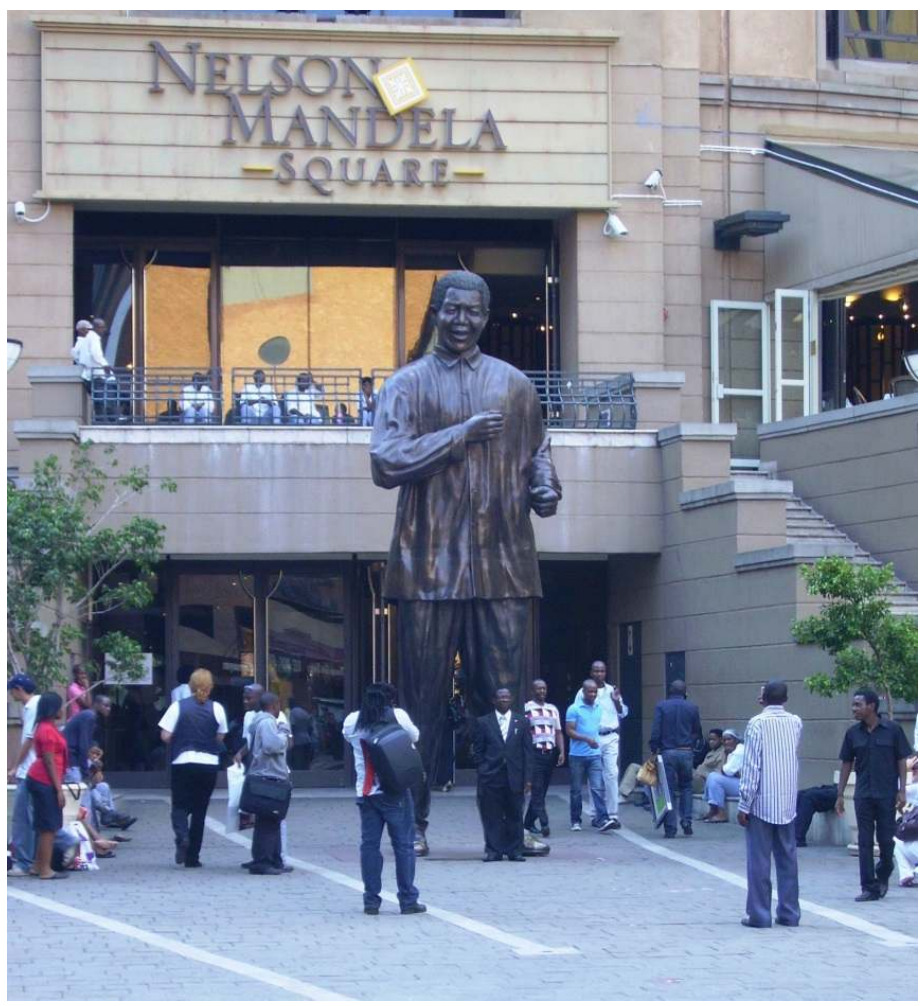

C P. GUINARD, 2009

Du fait de la renommée de Mandela dont le nom même tend à devenir un véritable label servant à promouvoir l'Afrique du Sud sur la scène internationale (Marschall, 2010a), la statue est pensée comme un moyen d'attirer des touristes et plus largement des publics nouveaux dans cet espace, alors que ce centre commercial - et plus largement Sandton était et est toujours largement perçu comme un espace blanc. La figure de Mandela, dans une sorte de mise en scène de l'Afrique du Sud post-apartheid, servirait alors à changer l'image de la place et du quartier, à l'« africaniser », de façon à accroître le nombre d'usagers, à diversifier les publics, et donc la clientèle, du centre commercial.

De la même façon, l'organisation régulière d'événements en tout genre sur cette place (concert gratuit à Pâques, dons de vêtements le jour de l'anniversaire de Mandela, etc.) est destinée à attirer un nombre toujours croissant d'usagers et de clients potentiels. En 
matière d'art, le partenariat public-privé des environs - le Sandton Central Management District (SCMD) - est même allé plus loin en développant à partir de 2005 un programme d'interventions artistiques intitulé Open Street Sessions (" Séances de rues ouvertes ") appelé à se déployer dans tous les espaces juridiquement publics de Sandton ainsi que dans l'espace juridiquement privé qu'est Mandela Square. Les objectifs de ce programme sont d'ailleurs conformes à ceux mobilisés pour justifier l'installation de la statue de Mandela. Comme l'art permanent, l'art temporaire est censé créer et vendre une certaine image du lieu en vue d'attirer une gamme de publics la plus large possible. Il est, en outre, aussi envisagé comme un moyen d'animer les espaces, d'y introduire de l'extraordinaire afin de séduire de nouveaux publics tout en évitant que les publics réguliers ne s'en lassent. Quelle que soit sa forme, l'art - en suscitant la venue de publics divers - peut donc être compris comme un vecteur potentiel de publicisation, notamment sociale, de l'espace. Mais, au-delà de ces intentions, comment la statue de Mandela et les différentes interventions artistiques qui ont lieu sur la place peuvent favoriser une publicisation sociale voire politique de cet espace pourtant juridiquement privé ?

$\mathrm{Si}$, par rapport à d'autres facteurs tels que la structure très diversifiée du marché de l'emploi de Sandton, l'émergence d'une classe moyenne noire, l'accessibilité et la centralité de l'espace ou bien encore sa sécurisation, l'art est certainement un facteur secondaire de diversification des publics de la place, son rôle dans les processus de publicisation à l'œuvre ne saurait être minoré. A cet égard, la statue de Mandela, par l'attention qu'elle suscite et les pratiques qu'elle génère, occupe une place particulière. En effet, venir à Mandela Square implique pour la majorité des visiteurs de prendre en photographie la statue et surtout de se faire prendre en photographie avec elle. A l'ère du téléphone portable qui a permis de démocratiser l'accès à la photographie, c'est la quasitotalité des usagers de cette place, et non uniquement les touristes ou les plus aisés, qui s'adonnent à cette pratique. Cet engouement entraîne alors une inévitable confrontation avec l'autre, qui peut être plus ou moins volontaire, amicale ou brève (éviter de passer devant la photographie des autres, attendre son tour pour poser devant la statue, etc.). Plus encore, le rapport à l'autre peut s'établir de manière délibérée, sur le mode de l'engagement volontaire et non contraint. Pour les personnes seules, mais également pour les couples, les familles ou les groupes qui désirent avoir une photographie de l'ensemble des personnes présentes, prendre une photographie avec Mandela nécessite le recours à un tiers. Dans ce cas, le meilleur candidat est généralement la première personne qui passe, et ceci indépendamment de sa couleur de peau, de sa catégorie sociale, de son âge ou de son genre, comme l'illustre cette scène ordinaire autour de la statue de Mandela :

Lundi 3 mars 2012. Midi. Un couple d'Indiens se fait prendre en photographie par un homme noir, seul. Un autre homme, également noir, passe par inadvertance derrière la statue à ce moment-là. Il s'excuse et se déplace. Le premier homme qui a pris la photographie pour le couple décide de se prendre lui aussi en photographie devant la statue, en tenant son téléphone à bout de bras. Cela fait sourire un autre passant, cette fois blanc, qui tape sur l'épaule du premier en passant à sa hauteur (Extrait de carnet de terrain de l'auteur).

La prise de photographies autour de la statue génère un ensemble de pratiques et de situations qui sont autant d'occasions de nouer un échange, même bref, même non verbal, avec l'autre. En tant que telle, la statue catalyse et augmente les opportunités de rencontres, et donc d'interactions entre les usagers de Mandela Square, favorisant ainsi la publicisation sociale de la place. 

le cadre du programme Open Street Sessions, dont nous ne pourrons analyser ici qu'un exemple, sont des situations privilégiées d'échange entre les différents usagers de la place. Le Water Ballet Divas («Ballet aquatique des divas») créé par Anthea Moys et Toni Morkel en 2008 est caractéristique des effets que ce type d'interventions a pu avoir sur la place et ses usagers. Conçu pour être parodique, ce ballet fut exécuté par six artistes sudafricains, blancs et noirs, tous non-professionnels de la danse. Sur fond de musique rock, les artistes, habillés en tenue de plage, une bouée autour de la taille, ont paradé sur la place, esquissant quelques gestes de danse maladroits, avant de poursuivre leur numéro dans la fontaine (figure 4).

Figure 4. Danser à Mandela Square pour faire des publics un public.

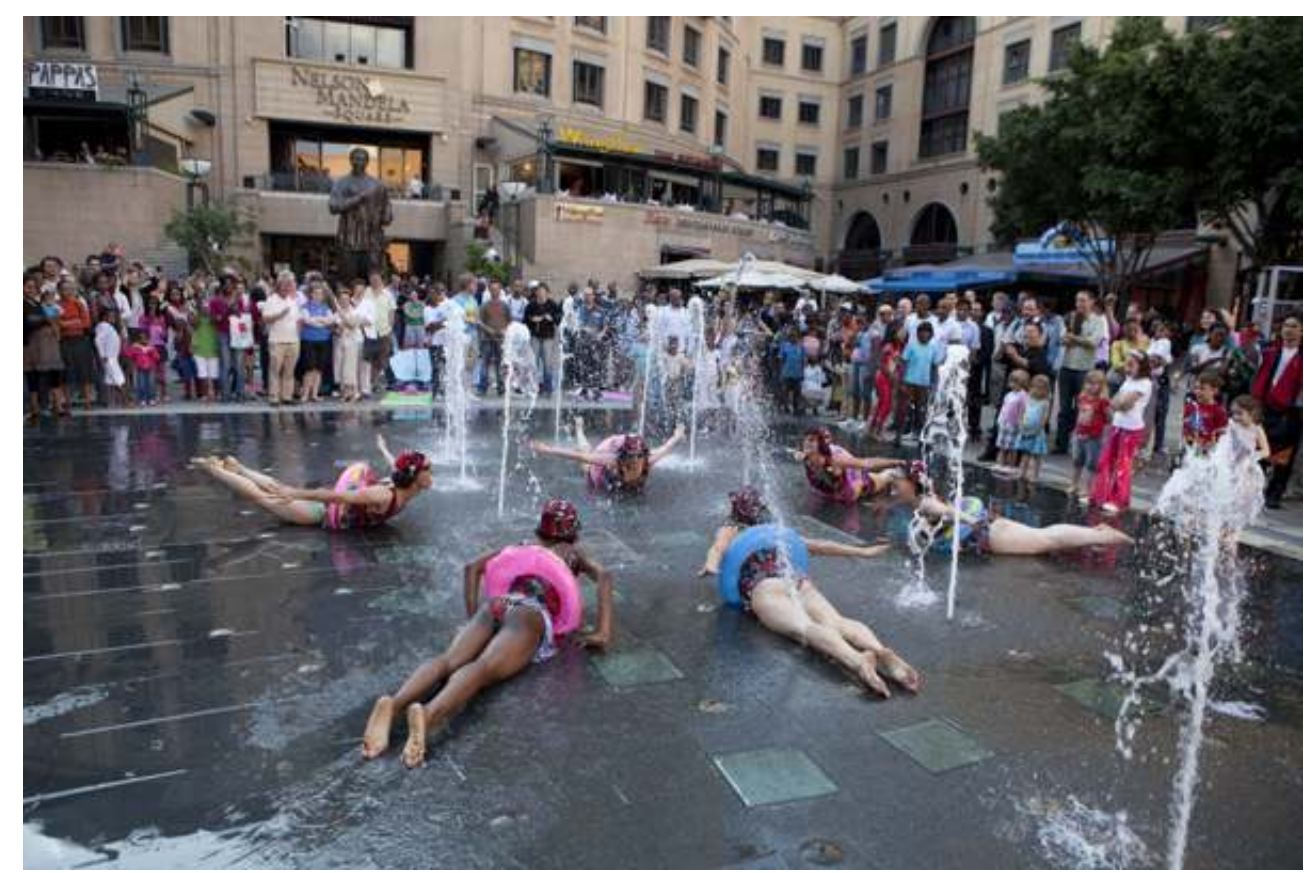

(c) Nadine Hutton et Chris Saunders, 2008, images reproduites avec l'autorisation d'A. Moys

31 Si nous n'avons pas nous-mêmes assisté à la scène, les différentes vidéos qui en ont été réalisées ${ }^{5}$ laissent entrevoir à quel point cette intervention a été l'occasion pour tous les usagers de la place de s'étonner, de rire en commentant les prouesses de tel ou tel artiste, de chanter ou bien encore de danser ensemble. En rompant avec le cours ordinaire de la vie de la place, en y introduisant une touche d'humour et de jeu, cette intervention a catalysé les échanges et les interactions entre ses usagers et leur a donné l'occasion de partager un instant et des émotions. Le concept de triangulation de William Whyte, qu'il définit comme un :

"process by which some external stimulus provides a linkage between people and prompts strangers to talk to other strangers as if they knew each other" $(1988$, p. 154)

est ici éclairant. Dans cette perspective, cette intervention peut être vue comme un «stimulus », c'est-à-dire un élément extérieur capable d'unir - certes temporairement les observateurs de cet événement dans une communauté de regard. L'acte de partager un événement favorise le rapprochement de personnes qui ne se connaissent pas, et ceci indépendamment de leurs différences individuelles. De ce rapprochement qui est à la fois 
symbolique et corporel peuvent découler des échanges verbaux ou non verbaux - ces deux dimensions des échanges étant tout aussi cruciales et constitutives des relations interpersonnelles (Daya, 2011) - entre des inconnus, qui ne constituent alors plus une somme d'individus isolés mais un ensemble de spectateurs. Les passants deviennent un public au sens social du fait qu'ils sont le public d'un spectacle. Les interventions comme celle-ci sont donc l'occasion, pour des gens qui habituellement se croisent sans nécessairement se rencontrer, de vivre une expérience commune.

Pour autant, en quoi un regard, un sourire ou des paroles échangés à l'occasion d'une intervention artistique ou d'une photographie peuvent-ils, au-delà de l'événement, transformer durablement le rapport à l'espace et à l'autre? Dans quelle mesure ces micro-événements peuvent-ils participer à la publicisation sociale, si ce n'est politique, de l'espace ? Comme l'a montré Isaac Joseph, le caractère limité - à la fois dans l'espace et dans le temps - de ces interactions ne suffit pas à les disqualifier, bien au contraire :

«Un événement microsociologique c'est toujours une aventure, dirait Simmel, quelque chose qui serait à la limite de l'essentiel et de l'accidentel. [...] L'essence se manifeste, non dans l'apparence, mais dans l'occasion» (1984, p. 54).

L'occasion d'un regard, d'un sourire ou d'une parole est constitutive de l'élaboration du rapport à l'autre dans les espaces publics. Si ces micro-événements comptent, c'est bien parce que ce qui est en jeu dans ces échanges, c'est la définition même des termes de la relation à l'autre, processus fondamental dans une société qui a longtemps été organisée selon une logique de séparation des différences, de mise à distance de l'altérité. Le simple fait que ces interactions, pour éphémères et limitées qu'elles soient, puissent avoir lieu, est déjà l'indice d'un engagement minimal avec l'autre, dans sa différence, et donc d'une certaine publicisation sociale de l'espace. Plus que cela, le jeu étant une incitation à imaginer des règles inédites de fonctionnement des choses et en l'occurrence des espaces, la dimension ludique d'une performance comme le Water Ballet Divas peut être l'occasion de tester, de contourner, voire de défier les normes établies, et de produire de nouvelles façons de se rapporter aux espaces et aux autres (Stevens, 2007 ; Moys, 2008). Parce que ces expériences sont communes, parce qu'elles incitent à la participation et sont ainsi des moments privilégiés de la construction d'une vie en société autre (Hawkins, 2011), elles sont aussi un facteur de publicisation politique des espaces. Bien que minimales, ces interactions participent constamment à l'invention du caractère socialement et politiquement public des espaces, et par là même à celle de la ville (Agier, 1999).

\section{Conclusion}

35 A partir du cas de Mandela Square, il est donc possible de mettre en évidence le rôle de l'art, permanent et temporaire, dans l'appréhension et dans la production des processus spatiaux à l'œuvre dans les espaces publics de Johannesburg. Parce qu'il permet de mettre en évidence et d'initier des mouvements de construction du caractère public de ces espaces, l'art peut être appréhendé comme un outil géographique à part entière, un prisme de lecture privilégié des espaces publics que l'on dit sans doute trop hâtivement absents de Johannesburg.

Pour autant, ces processus de construction de la publicité des espaces sont fragiles. La publicisation des espaces par le biais de l'art à Johannesburg, et plus largement dans les villes sud-africaines, ne saurait se faire du jour au lendemain, sans oscillation entre activation et remise en cause de leur publicité qu'elle soit juridique, sociale ou politique. 
La fin du programme Open Street Sessions à la suite d'un conflit entre les propriétaires du Mandela Square et le SCMD en 2010, qui portait précisément sur le statut juridique de cet espace, est à cet égard révélatrice du caractère réversible de ces processus. Arguant du statut juridiquement privé de la place, ses propriétaires ont progressivement rejeté hors de cet espace les Open Street Sessions, ce qui a d'ailleurs contribué à l'arrêt total du programme. Néanmoins, cela n'a pas marqué la fin de toutes les formes d'interventions artistiques à Mandela Square. Des concerts y sont notamment toujours proposés, mais ces interventions sont bien différentes de celles qui pouvaient être précédemment organisées. Loin de provoquer la rencontre des usagers, loin de remettre en cause les normes de la place, ces dernières sont au contraire destinées à créer une ambiance agréable, à rendre l'expérience commerciale plus plaisante (Guinard, 2014). L'art qui est actuellement produit à Mandela Square est donc avant tout envisagé comme un facteur de marchandisation de l'espace (Gervais-Lambony, 2012), plus que comme un vecteur de sa publicisation. Ce cas n'en souligne que davantage la pertinence d'une approche au prisme de l'art des espaces publics en termes de processus qui permet de s'affranchir d'une lecture fixiste et essentialiste de ces espaces et ainsi de mieux comprendre les espaces et avec eux la ville à l'œuvre à Johannesburg.

\section{BIBLIOGRAPHIE}

AGIER M. (1999), L'Invention de la ville : banlieues, townships, invasions et favelas, Paris, Editions des Archives Contemporaines.

ARDENNE M. (2002), Un art contextuel : création artistique en milieu urbain, en situation, d'intervention, de participation, Paris, Flammarion.

BEAVON K. (2004), Johannesburg: The Making and Shaping of the City, Pretoria, Unisa Press.

BÉNIT-GBAFFOU C., FABIYI S. et PEYROUX E. (dir.) (2009), Sécurisation des quartiers et gouvernance locale: enjeux et défis pour les villes africaines, Afrique du Sud, Kenya, Mozambique, Namibie, Nigeria, Paris, Johannesburg, Karthala IFAS.

BERDOULAY V., GOMES da COSTA P.C. et LOLIVE J. (dir.) (2004), L'espace public à l'épreuve : régressions et émergences, Pessac, Maison des sciences de l'homme d'Aquitaine.

BOURRIAUD N. (1998), Esthétique relationnelle, Dijon, les Presses du réel.

BREMNER L. (2010), Writing the City Into Being, Johannesburg, Fourthwall Books.

CAPRON G. et HASCHAR-NOÉ N. (dir.) (2007), L'espace public urbain : De l'objet au processus de construction, Toulouse, Presses Universitaires du Mirail.

CHIVALLON C., MARNE N. et PROST D. (1998), « Artefact de lieu et urbanité : Le centre commercial interrogé », Annales de la Recherche Urbaine, 78, pp. 28-37.

DAVIS M. (1997), City of quartz: Los Angeles, capitale du futur, Paris, Éd. la Découverte. 
DAWSON A. (2005), « Geography of Fear: Crime and the Transformation of Public Space in PostApartheid South Africa ", in LOW S. and SMITH N. (ed.), The Politics of Public Space, New York, Abingdon, Routledge, pp. 123-142.

DAYA S. (2011), « Performing place, mobility and identity in South Africa », Area, 43, 4, pp. 488-494.

DESSOUROUX C. (2003), « La diversité des processus de privatisation de l'espace public dans les villes européennes », Belgeo, 1, pp. 21-46.

DEUTSCHE R. (1998), Evictions: art and spatial politics, Cambridge, MIT Press.

DIRSUWEIT T. (2002), « Johannesburg: Fearful City? », Urban Forum, 13, 3, pp. 3.

DORIER-APPRILL E. et al. (2007), « Fragmentations », in DORIER-APPRILL E. et GERVAIS-LAMBONY P. (dir.), Vies citadines, Paris, Belin, pp. 15-57.

FLEURY A. (2007), Les espaces publics dans les politiques métropolitaines. Réflexions au croisement de trois expériences : de Paris aux quartiers centraux de Berlin et Istanbul, Thèse de doctorat, Université de Paris 1 Panthéon-Sorbonne.

FYFE N.R. et BANNISTER J. (1996), « City Watching: Closed Circuit Television Surveillance in Public Spaces ", Area, 28, 1, pp. 37-46.

GERVAIS-LAMBONY P. (2003), « Afrique du Sud, les temps du changement », Hérodote, 111, 4, pp. 81-98.

GERVAIS-LAMBONY P. (2012), Nostalgies citadines en Afrique Sud [en ligne], http:// www.espacestemps.net/document9459.html, consulté le 20 juin 2012.

GHORRA-GOBIN C. (dir.) (2001), Réinventer le sens de la ville : les espaces publics à l'heure globale, Paris, Montréal, Budapest, L'Harmattan.

GOSS J. (1993), «The "magic of the mall": an analysis of form, function and meaning of the mall in the contemporary retail built environment ", Annals of the Association of American Geographers, 83, 1, pp. 18-47.

GRÉSILLON B. (2014), Géographie de l'art, Paris, Economica.

GUILLAUME P. (2004), « La violence urbaine à Johannesburg. Entre réalité et prétexte », Geographica Helvetica, 3, pp. 188-198.

GUINARD P. (2014), Johannesburg : l'art d'inventer une ville, Rennes, Presses Universitaires de Rennes.

HALL T. et ROBERTSON I. (2001), « Public Art and Urban Regeneration: Advocacy, claims and critical debates ", Landscape Research, 26, 1, pp. 5-26.

HAWKINS H. (2011), « Dialogues and Doings: Sketching the Relationships Between Geography and Art: Dialogues and doings », Geography Compass, 5, 7, pp. 464-478.

HAWKINS H. (2013), For Creative Geographies: Geography, Visual Arts and the Making of Worlds, Routledge.

HOUSSAY-HOLZSCHUCH M. (2010), Crossing boundaries - tome 3 : Vivre ensemble dans l'Afrique du Sud post-apartheid, Habilitation à diriger des recherches, Université Paris 1 Panthéon-Sorbonne.

HOUSSAY-HOLZSCHUCH M. et TEPPO A. (2009), «A mall for all? Race and public space in postapartheid Cape Town », Cultural Geographies, 16, 3, pp. 351-379.

JACOBS J. (1977) [1961], The death and life of great American cities, London, Penguin Books. 
JOSEPH I. (1984), Le passant considérable : essai sur la dispersion de l'espace public, Paris, Librairie des Méridiens.

JOSEPH I. (1998), La ville sans qualités, La Tour d'Aigues, Editions de l'Aube.

KOHN M. (2004, Brave new neighborhoods : the privatization of public space, New York, Routledge.

LACY S. (ed.). (1995), Mapping the terrain: new genre public art, Seattle, Bay Press.

LÉVY J. et LUSSAULT M. (dir.) (2003), Dictionnaire de la géographie, Paris, Belin.

MARSCHALL S. (2002), Community mural art in South Africa, Pretoria, University of South Africa.

MARSCHALL S. (2010a), Landscape of Memory: Commemorative Monuments, Memorials and Public Statuary in Post-apartheid South-Africa, Leiden, Brill Academic Publishers.

MARSCHALL S. (2010b), « Articulating Cultural Pluralism through Public Art as Heritage in South Africa », Visual Anthropology, 23, 2, pp. 77.

MILES M. (1997, Art, Space and the City, London, New York, Routledge.

MINTY Z. (2006), « Post- apartheid public art in Cape Town: Symbolic reparations and public space. », Urban Studies, 43, 2, pp. 421-440.

MITCHELL D. (2003), The Right to the City: Social Justice and the Fight for Public Space, New York, Guilford Publications.

MITCHELL D. et STAEHELI L.A. (2007), The People's Property?: Power, Politics, and the Public, New York, Routledge.

MORANGE M. (2011), « Droit à la ville, néolibéralisation et État développemental au Cap », Justice Spatiale Spatial Justice, 3, [en ligne], http://jssj.org/media/public_space_vo3.pdf, consulté le 10 mai 2012.

MOYS A.J.D. (2008), Enacting play: performance within the public domain, Master of Fine Arts, University of Witwatersrand (Johannesburg).

NAVEZ-BOUCHANINE F. (2001), « De l'espace public occidental aux espaces publics non occidentaux », Villes en parallèle, 32, pp. 120-134.

OLIVIER de SARDAN J.P. (1995), « La politique du terrain : Sur la production des données en anthropologie », Les terrains de l'enquête, 1, pp. 71-112.

ROSE G. (2007), Visual Methodologies: An Introduction to the Interpretation of Visual Materials, London, Sage.

RUBY C. (2001), L'art public : un art de vivre la ville, Bruxelles, La lettre volée.

RUBY C. (2002), « L'art public dans la ville », EspaceTemps.net, [en ligne], [URL : http:// www.espacestemps.net/document282.html],consulté le 20 août 2010.

SABATIER B. (2007), « De l'impossible espace public à la publicisation des espaces privés », in CAPRON G. et HASCHAR-NOÉ N. (dir.), L'espace public urbain : de l'objet au processus de construction, Toulouse, Presses Universitaires Mirail, pp. 175-191.

SCHUERMANS N., LOOPMANS M. VANDENABEELE J. (2012), « Public space, public art and public pedagogy », Social \& Cultural Geography, 13, 7, pp. 675-682.

SHARP J., POLLOCK V. et PADDISON R. (2005), « Just art for a just city: Public art and social inclusion in urban regeneration. », Urban Studies, 42, 5-6, pp. 1001-1023. 
SORKIN M. (1992), Variations on a theme park: the new American city and the end of public space, New York, Hill and Wang, Farrar, Strauss and Giroux.

STAEHELI L.A. et MITCHELL D. (2006), « USA’s Destiny? Regulating Space and Creating Community in American Shopping Malls », Urban Studies, 43, 5-6, pp. 977-992.

STEVENS Q. (2007), The Ludic City: Exploring the Potential of Public Spaces, London, New York, Routledge.

VAN ESSCHE É. (dir.) (2007), Les formes contemporaines de l'art engagé : de l'art contextuel aux nouvelles pratiques documentaires, Bruxelles, La lettre volée.

VANDER GUCHT D. (2004), Art et politique : pour une redéfinition de l'art engagé, Bruxelles, Labor.

VOLVEY A. (2007), « Land Arts. Les fabriques spatiales de l'art contemporain », Travaux de l'Institut de Géographie de Reims, 129-130, pp. 5-27.

VOLVEY A. (2010, «Spatialités d'une land activité : le Land Art à travers l'œuvre de Christo et Jeanne-Claude ", Activité artistique et spatialité, Paris, Harmattan.

VOLVEY A. (2012), Transitionnelles géographies : Sur le terrain de la créativité artistique et scientifique, Habilitation à diriger des recherches, Université Lumière - Lyon II.

WHYTE W. H. (1988), City: rediscovering the center, New York, Doubleday.

ZEBRACKI M. (2012), Public Artopia: Art in Public Space in Question, Pallas Publications.

ZUKIN S. (1995), The cultures of cities, Cambridge, Oxford, Victoria, Blackwell Publishers.

\section{NOTES}

1. Pour signifier le caractère historiquement et socialement construit de la notion de " race ", nous utilisons ici des guillemets. Toutefois pour alléger le propos, nous n'y aurons plus recours par la suite. De la même façon, nous emploierons - sans pour autant y souscrire - les catégories raciales telles qu'elles ont été définies par le régime d'apartheid par le Population Registration Act de 1950 (Blancs, Noirs, Indiens/Asiatiques et Coloured) parce qu'elles ont déterminé et déterminent encore largement les aires d'habitation et certaines pratiques des populations, mais aussi parce qu'elles restent des catégories de perception et de représentation communément utilisées en Afrique du Sud.

2. Les murals sont des sortes de fresques. Héritiers des peintures rupestres et influencés par les murals américains et européens, les murals sud-africains constituent un genre artistique à part entière (Marschall, 2002).

3. L'Afrique du Sud et Johannesburg figurent parmi les espaces les plus violents au monde. A l'échelle nationale, le taux d'homicides volontaires en 2010 était estimé à 31,8 pour 100000 personnes par l'Office des Nations Unies sur les drogues et le crime, alors que le taux de viols déclarés s'élevait à 127,6 pour 100000 personnes en 2012 selon les services de police sudafricains. Si la peur des populations est donc bien nourrie par une violence réelle, elle résulte aussi d'une violence imaginée, faite des représentations de ces mêmes populations quant à cette violence (Dirsuweit, 2002 ; Guillaume, 2004).

4. « de créer un lieu de rassemblement où les touristes locaux et étrangers puissent contempler les événements qui ont mené à la création du miracle de la nation arc-en-ciel. »
$5 . \quad$ Voir
http://www.facebook.com/photo.php?
$\mathrm{v}=74364141285 \&$ set $=0.66732221314 \&$ type=3\&theater et http://www.youtube.com/watch?
v=JQvTKb4xlJc\&feature=player_embedded.

Belgeo, 3 | 2014 
6. «processus par lequel un stimulus extérieur fournit un lien entre les gens et encourage des inconnus à parler à d'autres inconnus comme s'ils se connaissaient. »

\section{RÉSUMÉS}

Johannesburg est réputée pour être une ville sans espaces publics (Lévy et Lussault, 2013). Et de fait, dans un contexte post-apartheid, la notion même d'espaces publics pose problème (HoussayHolzschuch, 2010) : d'une part, parce que les ségrégations passées et présentes tendent à en faire des lieux de séparation des publics, de division et d'exclusion, et d'autre part, parce que les taux de criminalité élevés et le fort sentiment d'insécurité renforcent leur sécurisation et leur privatisation. Or, depuis la fin de l'apartheid (1994), l'art est présenté par les différents acteurs de la ville comme un moyen de réinventer les espaces publics, en redéfinissant les rapports des différents publics aux différents espaces, et des publics entre eux. L'art serait-il alors un facteur potentiel de construction du caractère public des espaces? Nous permettrait-il, en tant que géographe, de mettre en lumière les processus d'élaboration de ces espaces que l'on dit - sans doute trop hâtivement - absents de Johannesburg? A partir du cas de Johannesburg et plus particulièrement de Mandela Square, nous montrerons que l'art peut être un outil géographique à part entière, permettant de mettre en évidence et de comprendre les processus de construction ou de déconstruction des espaces publics.

Johannesburg is known to be a city without public spaces (Lévy et Lussault, 2013). In a postapartheid context, the notion of public spaces is indeed problematic (Houssay-Holzschuch, 2010) : on the one hand, the past and current segregations have tended to turn them into places where people are set apart, and on the other hand, the high rates of criminality as well as the strong feeling of insecurity have reinforced the trend toward their securization and privatization. However, since the end of apartheid (1994), art is - according to various urban players - supposed to be a way of reinventing public spaces by redefining the relationships of the by-passers with these spaces and of the by-passers among themselves in those. But could art be a means of building the publicness of spaces? Could it be a tool for geographers in order to highlight the making of these spaces that are - surely too promptly - reported missing in Johannesburg? From the Johannesburg case study and the Mandela Square one in particular, I will argue that art can be a geographical tool so as to reveal and understand the making or unmaking of public spaces.

\section{INDEX}

Mots-clés : espaces publics, art, statue, performance, Johannesburg, Mandela Square, savoir géographique

Keywords : public spaces, art, statue, performance, Johannesburg, Mandela Square, geographical knowledge 


\section{AUTEUR}

\section{PAULINE GUINARD}

Maître de conférences à l'Ecole normale supérieure de Paris, UMR Lavue-Laboratoire Mosaïques, pauline.guinard@gmail.com 